\title{
Exosomal microRNA in plasma as a non-invasive biomarker for the recurrence of non-small cell lung cancer
}

\author{
HITOSHI DEJIMA, HISAE IINUMA, RIE KANAOKA, NORIYUKI MATSUTANI and MASAFUMI KAWAMURA
}

Department of Surgery, Teikyo University School of Medicine, Tokyo 173-0003, Japan

Received September 8, 2015; Accepted September 9, 2016

DOI: $10.3892 / 01.2017 .5569$

\begin{abstract}
Predictive biomarkers for the recurrence of non-small cell lung cancer (NSCLC) in patients who have received curative resection are important for cancer treatment. The functional microRNAs (miRNAs/miRs) in the exosomes of plasma and serum samples are of interest as stable and non-invasive biomarkers for recurrence in cancer patients. The aim of the present study was to clarify the usefulness of plasma exosomal miRNAs as biomarkers for the prediction of recurrence in NSCLC following curative resection. First, microarray-based expression profiling of miRNAs derived from exosomes in the plasma of 6 patients was employed to identify a biomarker that distinguishes between patients with and without NSCLC recurrence. In the miRNA microarray analyses, the exosomal miR-21 and miR-4257 levels of the NSCLC patients showed marked upregulation in those individuals with recurrence compared with those without recurrence and healthy individuals. These two miRNAs were thus selected as recurrence-specific biomarkers and their potential was evaluated in a separate cohort of 195 NSCLC patients. In comparison to the levels in 30 healthy individuals, exosomal miR-21 and miR-4257 levels showed a significant increase in the NSCLC patients $(\mathrm{P}<0.01)$. When evaluating the clinicopathological significance of these miRNAs, exosomal miR-21 showed a significant association with tumor size and tumor-node-metastasis (TNM) stage $(\mathrm{P}<0.05)$. Exosomal miR-4257 showed a significant association with histological type, lymphatic invasion and TNM stage $(\mathrm{P}<0.05)$. The disease-free survival (DFS) rates of high exosomal miR-21 patients were significantly worse than those of low exosomal miR-21 patients $(\mathrm{P}<0.05)$, and the DFS rates
\end{abstract}

Correspondence to: Dr Hisae Iinuma, Department of Surgery, Teikyo University School of Medicine, 2-11-1 Kaga, Itabashi, Tokyo 173-0003, Japan

E-mail: iinuma@med.teikyo-u.ac.jp

Abbreviations: RT-PCR, reverse transcription-polymerase chain reaction; NSCLC, non-small cell lung cancer

Key words: biomarker, exosome, non-small cell lung cancer, microRNA-21, microRNA-4257 of patients with high exosomal miR-4257 levels were significantly worse than those with low exosomal miR-4257 levels $(\mathrm{P}<0.01)$. In the Cox multivariate analysis, plasma exosomal miR-21 and miR-4257 expression showed a significance association with DFS $(\mathrm{P}<0.05)$. These results suggest that plasma exosomal miR-21 and mir-4257 expression has potential as a predictive biomarker for recurrence in NSCLC patients who have received curative resection.

\section{Introduction}

Lung cancer, mainly non-small cell lung cancer (NSCLC), is the leading cause of cancer-associated mortality globally. More than 133,000 new diagnoses and over 77,000 associated mortalities are expected to occur in 2015 in Japan (1). In spite of progress in NSCLC therapy, the prognosis for patients with NSCLC remains poor (2). Therefore, clarification of the biomarkers most likely to predict the recurrence of NSCLC after curative surgery is required to improve the outcome of NSCLC patients.

MicroRNAs (miRNAs/miRs) are one of the most compelling molecular markers in the diagnosis and prognosis of tumors $(3,4)$. miRNAs are short $(20-24 \mathrm{nt})$ non-coding RNAs that take part in the post-transcriptional regulation of gene expression in multicellular organisms by impacting mRNA stability and translation. miRNAs target protein-coding mRNAs at the post-transcriptional level by directly cleaving mRNA or by inhibiting protein synthesis. miRNA dysregulation has been demonstrated to be associated with the initiation and progression of cancer, indicating that miRNAs could be used as molecular biomarkers for cancer diagnostics and for predicting the likely prognosis $(5,6)$.

Recent evidence has indicated that such miRNAs have been identified in the plasma and serum, and are important as non-invasive biomarkers for cancer patients (7). In NSCLC, certain studies have shown the prognostic value of specific miRNAs in the plasma and serum (8-11). Furthermore, a focus has been placed on miRNAs that have also been identified in the exosome of plasma and serum in an unexpectedly stable form, which is protected from endogenous RNase activity (12-14). Exosomes are small membrane vesicles (30-100 nm) whose derivation lies in the luminal membranes of multivesicular bodies and which are released by fusion with the cell membrane (15). It is known that exosomes transfer not only membrane components, but also nucleic acids such as 
miRNAs to other cells (16). Therefore, the exosomal transport of miRNAs has received attention in cancer research as a carrier of genetic information $(17,18)$. The early diagnosis of cancer is extremely important for improving the prognosis. However, the existing biomarkers, such as cancer-associated antigens, and proteomics analysis are unsatisfactory for the early stages of NSCLC. By contrast, recent studies have demonstrated that specific expression profiles of circulating exosomal miRNAs could be promising biomarkers for the detection of early-stage cancer and for predicting prognosis in various types of cancers (19-21). However, previous studies in NSCLC have not examined exosomal miRNAs, which could be expected to include the intact miRNA.

The present study clarified the potential of plasma exosomal miRNA as a biomarker for recurrence in NSCLC patients who have undergone curative surgery.

\section{Patients and methods}

Study design. In this study, 201 NSCLC patients were included. First, the recurrence-specific exosomal miRNAs of 6 NSCLC patients were profiled by miRNA array using a 3D-Gene Human miRNA Oligo chips ver.20 (Toray Industries, Inc., Kamakura, Japan). Next, the usefulness of selected miRNAs as biomarkers was clarified using another 195 NSCLC cases. This study was approved by the Institutional Review Board of the Ethics and Indications Committee of Teikyo University (Tokyo, Japan). Written informed consent was obtained from all patients. All patients underwent curative surgery, and the median follow-up period was 20 months (range, 0.5-37 months). NSCLC patients were diagnosed according to the current World Health Organization histological classification and were staged according to the TNM classification of the International Union Against Cancer $(22,23)$. Patients were treated with a standard regimen (uracil/tegafur for TNM stage I patients and cisplatin plus vinorelbine for TNM stage II and III patients) in accordance with the Guidelines of the Japan Lung Cancer Society (24). Post-operative follow-up was performed as follows: Confirmation of recurrence in all patients was required to evaluate imaging or pathological diagnosis. Physical examination and chest X-ray were conducted every 3 months for 5 years, and computed tomography was repeated every 6 months for 5 years after surgery.

Profiling study. For recurrence-specific miRNA microarray profiling, 6 cases of NSCLC (TNM stage III) were selected from the records of the Department of Surgery, Teikyo University. Tumor recurrence was found in 3 cases of the 6 cases after surgery. Recurrence sites identified were the brain ( 2 cases) and the bones ( 1 case). As a normal control, blood samples from 3 healthy volunteers were examined. All blood samples were derived from patients who had not received any chemotherapy or radiotherapy prior to surgery. Plasma samples were separated from the blood, and exosomes were purified from the plasma samples. Exosomal miRNA expression profiles were examined using a 3D-Gene Human miRNA Oligo chips ver.20 (Toray Industries, Inc., Kamakura, Japan). Details of the measuring methods are described as follows.
Validation study. For the validation analysis, 195 consecutive NSCLC patients and 30 healthy individuals treated between April 2012 and July 2015 at Teikyo University Hospital (Tokyo, Japan) were studied. These NSCLC patients included 129 cases with tumor-node metastases (TNM) stage I, 34 cases with stage II and 32 cases with stage III. All blood samples were derived from patients who had not received any chemotherapy or radiotherapy prior to surgery. Plasma samples were separated from the blood, and exosomes were purified from the plasma samples. Exosomal miRNA expression was analyzed using reverse transcription-quantitative polymerase chain reaction (RT-qPCR). Details of the measuring method are described as follows.

Purification of exosomes from plasma. A peripheral blood specimen $(5 \mathrm{ml})$ was drawn from each patient prior to surgery. Ethylenediaminetetraacetic acid was used as an anticoagulant. Plasma and blood cells were separated by centrifugation at $1,200 \times \mathrm{g}$ for $10 \mathrm{~min}$ at $4^{\circ} \mathrm{C}$. Plasma $(1.0 \mathrm{ml})$ was used for microarray analysis, and $500 \mu \mathrm{l}$ was used for RT-qPCR. The exosomes of the plasma were purified by the ultracentrifugation method, as described previously (21). In brief, the exosomes were separated by ultracentrifugation at 100,000 x $g$ for $70 \mathrm{~min}$ at $4^{\circ} \mathrm{C}$, and then the pellets were washed with phosphate-buffered saline (PBS) and stored at $-80^{\circ} \mathrm{C}$ for microarray and RT-qPCR analysis.

Transmission electron microscopy of exosomes. The isolated exosomes were dissolved in PBS, and a drop of the suspension was placed on a carbon-coated copper grid for $10 \mathrm{sec}$. The grid was then removed and excess liquid was drained by filter paper. The grid was put in contact with a drop of $2 \%$ uranyl acetate and phosphotungstic acid for $5 \mathrm{sec}$, and excess liquid was then removed. The grid was allowed to dry for several min and was then examined using an electron microscope (HITACHI H-7600; Hitachi Ltd., Tokyo, Japan) at $100 \mathrm{kV}$.

miRNA isolation from exosomes. RNA was isolated from exosomes using the miRNeasy serum/plasma kit (Qiagen, Hilden, Germany). Exosomes purified from specific volumes of plasma $(1.0 \mathrm{ml}$ for microarray analysis and $500 \mu \mathrm{l}$ for RT-qPCR assay) were diluted with $1 \mathrm{ml}$ Qiazol Lysis Reagent. Following incubation for $5 \mathrm{~min}, 3.5 \mu \mathrm{l}$ of a spike-in control (cel-miR-39 mimic) was added to each sample. Subsequent extraction and cartridge work were performed according to the manufacturer's protocols. The RNA quality was assessed using an Agilent 2100 Bioanalyzer (Agilent Technologies, Inc., Santa Clara, CA, USA).

miRNA microarray analysis. Exosomal miRNA expression profiles were examined using 3D-Gene Human miRNA Oligo chips ver. 20 (Toray Industries, Inc. Tokyo, Japan), according to the manufacturer's protocol. Fluorescence signals were scanned and analyzed using the 3D-Gene Scanner (Toray Industries, Inc.). A total of 2578 genes were mounted on this chip. The raw data from each spot were normalized by subtraction of the background signal mean intensity, determined by the $95 \%$ confidence intervals of the signal intensities of all blank spots. Valid measurements were considered those in which the signal intensity of the 
Table I. Clinicopathological factors of patients used for the microRNA array.

\begin{tabular}{lcc}
\hline $\begin{array}{c}\text { Patients with } \\
\text { recurrence after } \\
\text { surgery }(n=3)\end{array}$ & $\begin{array}{c}\text { Patients without } \\
\text { recurrence after } \\
\text { surgery }(n=3)\end{array}$ \\
\hline
\end{tabular}

Tumor size, $\mathrm{cm}$

$\begin{array}{lll}<3 & 0 & 0 \\ \geqq 3 & 3 & 3\end{array}$

Lymph node metastasis

$\mathrm{n}(-)$

$\begin{array}{ll}0 & 0 \\ 3 & 3 \\ & \\ 0 & 0 \\ 3 & 3\end{array}$

Lymphatic invasion

Ly (-)

Ly (+)

Venous invasion

$\mathrm{V}(-)$

$\mathrm{V}(+)$

0

0 3 3

Histological type

Adenocarcinoma

Squamous cell carcinoma

2

2

Tumor differentiation

\begin{tabular}{lll} 
Well & 0 & 0 \\
Moderate & 2 & 2 \\
Poor & 1 & 1 \\
TNM stage & & \\
I & 0 & 0 \\
II & 0 & 0 \\
III & 3 & 3 \\
\hline
\end{tabular}

TNM, tumor-node-metastasis.

duplicate spots was $>2$ standard deviations of the background signal intensity.

RT-qPCR for miRNAs. Exosomal miRNA expression was assayed using RT-qPCR. cDNA was synthesized from total RNA using Taqman microRNA primers specific for hsa-miR-21 (assay ID 000397), has-miR-4257 (assay ID 244369) and has-miR-16a (assay ID 000391) (Thermo Fisher Scientific, Inc., Waltham, MA, USA) and a TaqMan Micro-RNA Reverse Transcription kit (Thermo Fisher Scientific, Inc.). miR-16a was used as an internal control, as it is reported to be a reliable endogenous control for miRNA analysis in RT-qPCR for humans (21). RT-qPCR was performed using Lightcycler-480 (Roche Applied Science, Basel, Switzerland) and Taqman Universal PCR Master Mix (Thermo Fisher Scientific, Inc.) following the manufacturer's protocols. Relative quantification of miRNA expression was calculated using the $2^{-\Delta \Delta \mathrm{Cq}}$ method (25). $\Delta \mathrm{Cq}$ was calculated by subtracting the $\mathrm{Cq}$ values of miR-16 from the $\mathrm{Cq}$ values of miR-21 or miR-4257. $\Delta \Delta \mathrm{Cq}$ was then calculated by subtracting the $\Delta \mathrm{Cq}$ of the healthy control from the $\Delta \mathrm{Cq}$ of the sample. For healthy controls, plasma exosome samples of 30 healthy volunteers were used.

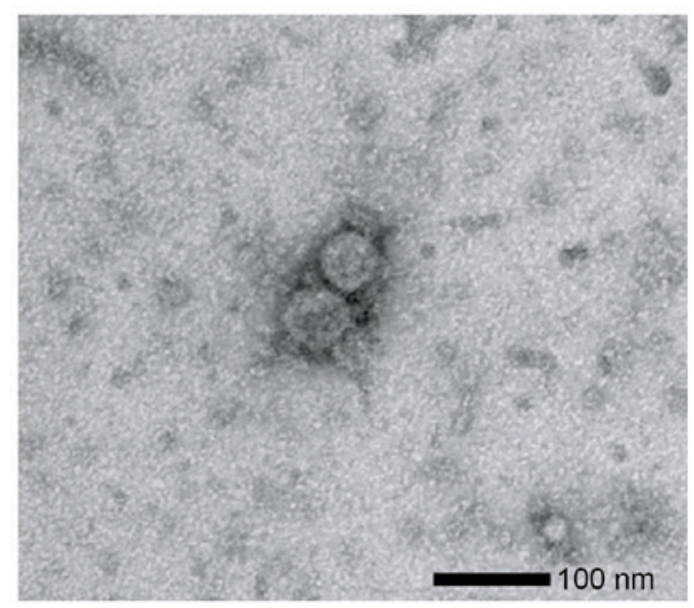

Figure 1. Exosome images captured by transmission electron microscope. Original magnification, $\mathrm{x} 25,000$.

Statistical analysis. The data are expressed as the mean \pm standard deviation. The correlations between microRNA expression and clinicopathological factors were analyzed using Student's $t$-test, the $\chi^{2}$ test and an analysis of variance. Disease-free survival (DFS) curves were analyzed using the Kaplan-Meier survival curve method, and the differences were examined using log-rank tests. Cox proportional-hazards regression analysis was used to estimate univariate and multivariate hazard ratios for DFS. All P-values are two-sided, and $\mathrm{P}<0.05$ was considered to indicate a statistically significant difference. Statistical analyses were performed using the JMP 9.0 software (SAS Institute, Inc., Tokyo, Japan).

\section{Results}

Identification of exosomes in plasma. To verify the isolation of the exosomes from the plasma, transmission electron microscopy results were examined of ultracentrifugation samples from the NSCLC patients. Images of round microvesicles with diameters of 50-100 $\mathrm{nm}$ were captured, as shown in a representative sample in Fig. 1.

Exosomal miRNA profile of NSCLC patients. To identify the recurrence-specific miRNA profiling in the plasma exosomes of the NSCLC patients, the miRNA microarray analyses of samples from 3 patients with recurrence after surgery, 3 patients without recurrence after surgery and 3 healthy volunteers were examined. The clinical pathological characteristics of the 6 NSCLC patients used for this analyses are shown in Table I. In these patients, the histological type and the TNM stages were the same in the recurrence and recurrence-free cases. In the 3 recurrence cases, the recurrent sites were the brain ( 2 cases) and the bones (1 case).

Table II shows the five most highly upregulated exosomal miRNAs in the patients with recurrence. In this analysis, miR-4257 (miRBase no. MIMAT0016878) showed the highest upregulation in the patients with recurrence compared with those without recurrence and the healthy controls. The mean fold-change of miR-4257 was 2.1 in comparison with that of the recurrence-free patients, and 2.9 in comparison with that of the healthy controls. miR-21 
Table II. Five most highly upregulated miRNAs in the non-small cell lung cancer patients with recurrence based on the miRNA profiling array.

\begin{tabular}{lllcr}
\hline Ranking & miRNA & miRBase no. & $\begin{array}{c}\text { Fold-change (comparison with } \\
\text { recurrence-free patients) }\end{array}$ & $\begin{array}{c}\text { Fold-change (comparison } \\
\text { with healthy controls) }\end{array}$ \\
\hline 1 & miR-4257 & MIMAT0016878 & 2.1 & 2.9 \\
2 & miR-21 & MIMAT0000076 & 1.7 & 2.5 \\
3 & miR-887 & MIMAT0004951 & 1.4 & 1.9 \\
4 & miR-6794 & MIMAT0027488 & 1.3 & 1.8 \\
5 & miR-4476 & MIMAT0019003 & 1.3 & 1.7 \\
\hline
\end{tabular}

miR/miRNA, microRNA.
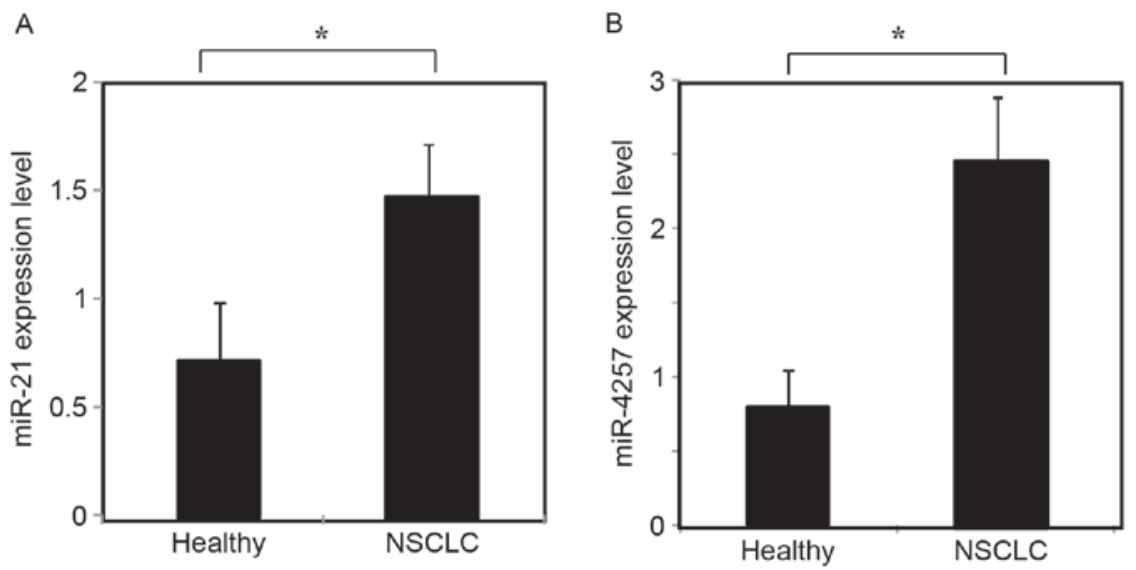

Figure 2. Expression of miR-21 and miR-4257 in NSCLC patients and healthy individuals. To investigate the miRNA expression of exosome samples, reverse transcription-quantitative polymerase chain reaction using Taqman miRNA assays was performed. (A) Comparison of exosomal miR-21 levels between the 30 healthy volunteers and 195 patients with NSCLC. Exosomal miR-21 levels showed a significant increase in the NSCLC patients ("P<0.01). (B) Comparison of exosomal miR-4257 levels between the 30 healthy volunteers and 195 patients with NSCLC. Exosomal miR-4257 levels showed a significant increase in the NSCLC patients ( $\mathrm{P}<0.01)$. miR/miRNA, microRNA; NSCLC, non-small cell lung cancer.

(miRBase no. MIMAT0000076) showed the highest level after miR-4257. The mean fold-change of miR-21 was 1.7 in comparison with that of recurrence-free patients, and 2.5 in comparison with that of the healthy controls. On the basis of these profiling results, miR-4257 and miR-21 were selected as the recurrence markers for NSCLC cases and were used in the present study.

Expression of exosomal miR-21 and $m i R-4257$. The expression of miR-21 and miR-4257 was assessed by RT-qPCR in the plasma exosomal samples from 195 patients with NSCLC and 30 healthy volunteers. As shown in Fig. 2A and B, exosomal miR-21 and miR-4257 levels were significantly increased in NSCLC patients as compared with those of healthy controls $(\mathrm{P}<0.01)$.

Clinicopathological significance of $\mathrm{miR}-21$ and $\mathrm{miR}-4257$ expression in plasma exosomes. This study includes 195 NSCLC patients (112 men and 83 women), with a mean age of 71 years (range, $32-88$ years). These patients were drawn from a separate cohort to the miRNA microarray study. To evaluate the correlation between the exosomal miR-21 and miR-4257 expression levels and the clinicopathological characteristics, patients were divided into two groups (high and low). The cut-off level was determined as the average of the miR-21 and miR-4257 expression levels, as described previously (21). As shown in Table III, a statistically significant association was observed between the high miR-21 group and tumor size and TNM stage. A statistically significant association was observed between the high miR-4257 group and lymphatic invasion, histological type and TNM stage (Table IV).

Correlation between miR-21 and miR-4257 levels and DFS. The Kaplan-Meier DFS curves of the 195 NSCLC patients (TNM stage I, 129 cases; TNM stage II, 34 cases; TNM stage III, 32 cases) according to the status of miR-21 and miR-4257 levels were examined. Patients who had undergone curative surgery were included in this analysis.

The DFS of patients in the high miR-21 group showed a significantly worse survival rate than those in the low miR-21 group (log-rank, $\mathrm{P}=0.016$; Fig. 3). The DFS analysis revealed that patients in the high miR-4257 group exhibited significantly worse survival rates than those in the low miR-4257 group (log-rank, $\mathrm{P}=0.005$; Fig. 4). These results suggest that the high expression of miR-21and miR-4257 is associated with recurrence in NSCLC patients. 
Table III. Association between clinicopathological factors and high exosomal miR-21 expression.

\begin{tabular}{|c|c|c|c|c|}
\hline Factors & No. of patients $(n=195)$ & miR-21 high $(n=88)$ & Positive rate, $\%$ & P-value \\
\hline Gender & & & & 0.65 \\
\hline Male & 112 & 49 & 43.8 & \\
\hline Female & 83 & 39 & 47.0 & \\
\hline Smoking status & & & & 0.11 \\
\hline Non-smokers & 68 & 36 & 52.9 & \\
\hline Smokers & 127 & 52 & 40.9 & \\
\hline Tumor size, $\mathrm{cm}$ & & & & 0.02 \\
\hline$<3$ & 136 & 54 & 39.7 & \\
\hline$\geq 3$ & 59 & 34 & 57.6 & \\
\hline Lymph node metastasis & & & & 0.10 \\
\hline n (-) & 148 & 59 & 39.9 & \\
\hline $\mathrm{n}(+)$ & 47 & 29 & 61.7 & \\
\hline Lymphatic invasion & & & & 0.29 \\
\hline Ly (-) & 153 & 66 & 43.1 & \\
\hline $\operatorname{Ly}(+)$ & 42 & 22 & 52.4 & \\
\hline Venous invasion & & & & 0.56 \\
\hline $\mathrm{V}(-)$ & 122 & 57 & 46.7 & \\
\hline $\mathrm{V}(+)$ & 73 & 31 & 42.5 & \\
\hline Histological type & & & & 0.87 \\
\hline Adenocarcinoma & 134 & 60 & 44.8 & \\
\hline Squamous cell carcinoma & 53 & 25 & 47.2 & \\
\hline Others & 8 & 3 & 37.5 & \\
\hline Tumor differentiation & & & & 0.43 \\
\hline Well & 91 & 37 & 40.7 & \\
\hline Moderate & 74 & 35 & 47.3 & \\
\hline Poor & 30 & 16 & 53.3 & \\
\hline TNM stage & & & & 0.03 \\
\hline I, II & 163 & 68 & 41.7 & \\
\hline III & 32 & 20 & 62.5 & \\
\hline
\end{tabular}

miR/miRNA, microRNA; TNM, tumor-node-metastasis.

Univariate and multivariate Cox analysis for DFS. Table V shows the results of univariate and multivariate Cox proportional hazard regression analysis for DFS in NSCLC patients $(n=195)$. Multivariate analysis was performed for all factors tested by univariate analysis. In the univariate analysis, tumor size, lymphatic invasion, TNM stage, and miR-21 and miR-4257 levels showed significance for DFS. In the multivariate analysis, TNM stage, and miR-21 and miR-4257 levels showed significance for DFS. These results suggest that miR-21 and miR-4257 levels in tumor tissues have an independent prognostic value for DFS in NSCLC patients.

\section{Discussion}

The aim of the present study was to clarify the specific plasma exosome miRNAs as biomarkers reflecting the recurrence of NSCLC. It was found that plasma exosomal miR-21 and miR-4257 could be potential biomarkers to predict recurrence in NSCLC.
Recent studies showed that miRNAs are preserved in a stable form in the exosome, are protected from endogenous RNase activity and are important in the cell to cell information processes $(15,16)$. In the preliminary experiments of the present study, the expression levels of miR-21 were compared between the plasma and exosome samples separated from plasma in the same NSCLC patients. It was found that the expression of exosomal RNA was at high and stable levels as compared with that of the plasma samples (data not shown). This may be due to the breakdown of the miRNA in the plasma samples. However, the majority of the previous studies in NSCLC did not examine the exosomal miRNAs separated from the plasma or serum, which would likely include the intact miRNA (7-13,26-29). In the present study, microarray-based expression profiling of miRNAs derived from exosomes in the plasma of 6 NSCLC patients with or without recurrence was employed. In the microarray analysis, miR-4257 and miR-21 showed marked upregulation in the NSCLC patients with recurrence after surgery compared with those without 
Table IV. Association between clinicopathological factors and high exosomal miR-4257 expression.

\begin{tabular}{|c|c|c|c|c|}
\hline Factors & No. of patients $(n=195)$ & miR-4257 high $(n=54)$ & Positive rate, $\%$ & P-value \\
\hline \multicolumn{5}{|l|}{ Gender } \\
\hline Male & 112 & 29 & 25.9 & 0.51 \\
\hline Female & 83 & 25 & 30.1 & \\
\hline \multicolumn{5}{|l|}{ Smoking status } \\
\hline Non-smokers & 68 & 20 & 29.4 & 0.60 \\
\hline Smokers & 127 & 34 & 26.8 & \\
\hline \multicolumn{5}{|l|}{ Tumor size, $\mathrm{cm}$} \\
\hline$<3$ & 136 & 35 & 25.7 & 0.35 \\
\hline$\geqq 3$ & 59 & 19 & 32.2 & \\
\hline \multicolumn{5}{|l|}{ Lymph node metastasis } \\
\hline n (-) & 148 & 36 & 24.3 & 0.06 \\
\hline $\mathrm{n}(+)$ & 47 & 18 & 38.3 & \\
\hline \multicolumn{5}{|l|}{ Lymphatic invasion } \\
\hline Ly (-) & 153 & 37 & 24.2 & 0.04 \\
\hline Ly (+) & 42 & 17 & 40.5 & \\
\hline \multicolumn{5}{|l|}{ Venous invasion } \\
\hline V (-) & 122 & 35 & 28.7 & 0.69 \\
\hline $\mathrm{V}(+)$ & 73 & 19 & 26.0 & \\
\hline \multicolumn{5}{|l|}{ Histological type } \\
\hline Adenocarcinoma & 134 & 47 & 35.1 & 0.02 \\
\hline Squamous cell carcinoma & 53 & 5 & 9.4 & \\
\hline Others & 8 & 2 & 25.0 & \\
\hline \multicolumn{5}{|l|}{ Tumor differentiation } \\
\hline Well & 91 & 26 & 28.6 & 0.97 \\
\hline Moderate & 74 & 20 & 27.0 & \\
\hline Poor & 30 & 8 & 26.7 & \\
\hline \multicolumn{5}{|l|}{ TNM stage } \\
\hline I, II & 163 & 38 & 23.3 & $<0.01$ \\
\hline III & 32 & 16 & 50.0 & \\
\hline
\end{tabular}

miR/miRNA, microRNA; TNM, tumor-node-metastasis.

Table V. Univariate and multivariate analyses of the prognostic factors for disease-free survival in all patients.

\begin{tabular}{|c|c|c|c|c|c|c|}
\hline \multirow[b]{2}{*}{ Factors } & \multicolumn{3}{|c|}{ Univariate analysis } & \multicolumn{3}{|c|}{ Multivariate analysis } \\
\hline & $\begin{array}{l}\text { Regression } \\
\text { coefficient }\end{array}$ & $\begin{array}{l}\text { Hazard ratio } \\
(95 \% \mathrm{CI})\end{array}$ & P-value & $\begin{array}{c}\text { Regression } \\
\text { coefficient }\end{array}$ & $\begin{array}{l}\text { Hazard ratio } \\
(95 \% \mathrm{CI})\end{array}$ & P-value \\
\hline Smoking status & 0.75 & $2.11(0.64-9.43)$ & 0.229 & 0.05 & $1.05(0.24-5.55)$ & 0.950 \\
\hline Tumor size & 1.65 & $5.20(1.73-17.27)$ & 0.004 & 0.76 & $2.13(0.58-8.21)$ & 0.254 \\
\hline Lymphatic invasion & 1.29 & $3.63(1.18-11.59)$ & 0.025 & 0.83 & $2.30(0.71-7.65)$ & 0.163 \\
\hline Venous invasion & 0.98 & $2.66(0.79-8.04)$ & 0.107 & 0.32 & $1.37(0.24-6.89)$ & 0.708 \\
\hline Histological type & 0.73 & $2.07(0.66-6.25)$ & 0.203 & 0.46 & $1.58(0.31-7.88)$ & 0.576 \\
\hline Tumor differentiation & 0.83 & $2.29(0.74-8.51)$ & 0.152 & -0.01 & $0.99(0.25-4.55)$ & 0.991 \\
\hline TNM stage & 2.59 & $13.35(4.09-51.78)$ & $<0.001$ & 2.07 & $7.96(1.97-36.09)$ & 0.004 \\
\hline $\operatorname{miR}-21$ & 1.37 & $3.93(1.26-14.65)$ & 0.018 & 1.34 & $3.81(1.13-14.93)$ & 0.031 \\
\hline $\operatorname{miR}-4257$ & 1.98 & $7.27(2.21-32.49)$ & 0.001 & 2.09 & $8.09(2.41-36.73)$ & 0.001 \\
\hline
\end{tabular}

miR/miRNA, microRNA; TNM, tumor-node-metastasis; CI, confidence interval. 


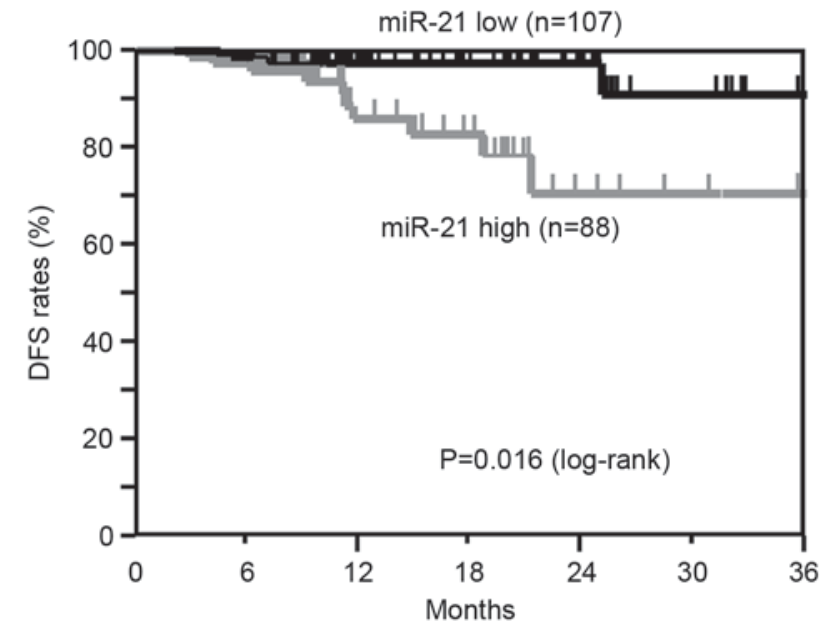

Figure 3. Kaplan-Meier survival curves of DFS based on exosomal miR-21 expression in non-small cell lung cancer patients. Patients were divided into two groups: those with low and those with high miR-21. The significance of these groups with regard to DFS was demonstrated. miR, microRNA; DFS, disease-free survival.

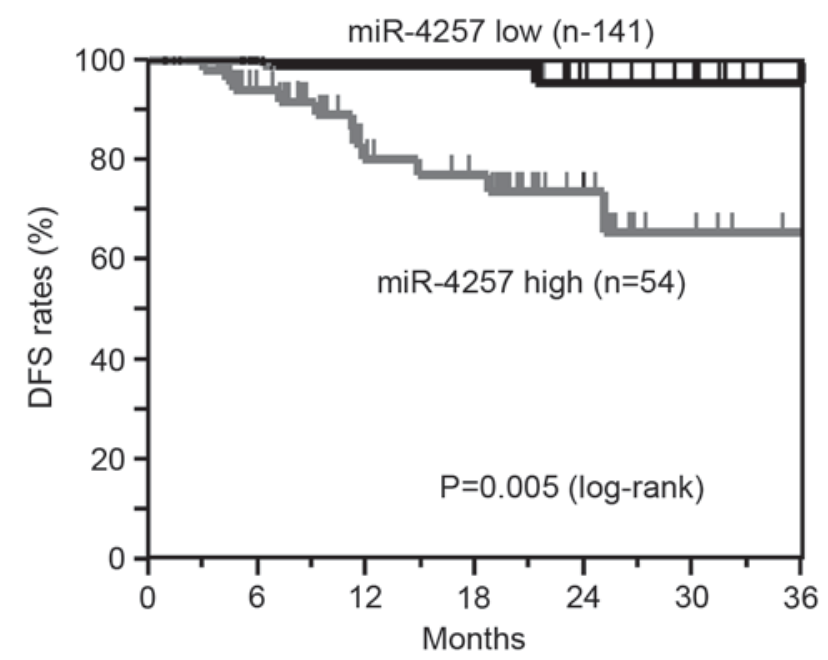

Figure 4. Kaplan-Meier survival curves of DFS based on exosomal miR-4257 expression in non-small cell lung cancer patients. Patients were divided into two groups: those with low and those with high miR-4257. The significance of these groups for DFS was demonstrated. miR, microRNA; DFS, disease-free survival.

recurrence after surgery. Furthermore, these two exosomal miRNAs levels were markedly increase as compared with those of the healthy controls. miR-4257 showed the highest level of all miRNAs, with miR21 showing the second highest level. From these results, miR-4257 and miR-21 were selected as recurrence-specific biomarkers for NSCLC patients.

It has been reported that serum miR-21 is significantly associated with the promotion of tumor growth, proliferation and progression, and the response of cancer to chemotherapy (26). In an evaluation of clinicopathological significance, Wang et al reported that serum miR-21 expression was significantly correlated with advanced TNM stage and the presence of lymph node metastasis in NSCLC patients (27). However, the status of plasma exosomal miR-21 expression and its potential as a biomarker in NSCLC remains unclear. In the present study, using the exosomal samples separated from the plasma of 195 NSCLC patients, it was shown that high miR-21 expression has a significant association with tumor size and TNM stage. By contrast, miR-4257 is a novel microRNA and the association of clinicopathological factors in NSCLC patients has not been hitherto reported. To the best of our knowledge, the present study is the first to show that exosomal miR-4257 levels are significantly associated with lymphatic invasion, histological type and TNM stage.

The prognostic value of the miRNA of serum or plasma samples as non-invasive biomarkers in NSCLC patients has been reported. Sanfiorenzo et al showed the importance of the two panels of plasma miRNAs for the prediction of recurrence in NSCLC patients (28). The study reported that one miRNA panel (high miR-155-5p, high miR-223-3p and low miR-126a-3p) was significantly associated with a higher progression risk in adenocarcinoma patients, and that another miRNA panel (high miR-20a-5p, low miR-152-3p and low miR-199a-5p) was significant in the prediction of likely survival rates of squamous cell carcinoma patients. Gao et al reported that the miR-155 overexpression in serum specimens may be a diagnostic marker for the early detection of lung adenocarcinoma (29). The clinical significance of serum miR-21 expression, but not exosomes, has also been reported. Liu et al demonstrated that serum miR-21 and tumor miR-21, miR-141 and miR-200c may be potential biomarkers for the diagnosis of NSCLC (30). Wang et al showed that serum miR-21 expression may be useful as a prognostic marker for NSCLC patients (27).

Using a separate cohort of 195 NSCLC patients who underwent curative surgery, the present study investigated the prognostic significance of plasma exosomal miR-21 and miR-4257 levels using the Kaplan-Meier method and Cox multivariate analysis. It was demonstrated that plasma exosomal miR-21 expression is a potential predictive biomarker for recurrence in NSCLC patients. Furthermore, the data showed that plasma exosomal miR-4257 also has an independent prognostic impact on DFS in NSCLC patients. To the best of our knowledge, this is the first study to clarify the significant prognostic value of exosomal miR-4257 in NSCLC patients.

miRNAs target protein-coding mRNAs at the post-transcriptional level by directly cleaving the mRNA in two different manners, namely, via directly cleaving the target mRNAs and by inhibiting protein synthesis (31). Bioinformatically predicted targets for miR-21 that have been experimentally validated include the tumor suppressor genes programmed cell death 4 (PDCD4), phosphatase and tensin homolog, tropomypsin 1 and reversion-inducing cysteine-rich protein (32-34). PDCD4 has been characterized as a novel tumor suppressor gene that acts as a suppressor of transformation, progression, invasion and metalloproteinase activation, and as an inducer of apoptosis. We have previously demonstrated a significant inverse association between miR-21 and PDCD4 mRNA in colorectal cancer patients (34). Through the inhibition of the PDCD4 tumor suppressor gene by miR-21, tumor growth may be promoted, leading to a poor prognosis. By contrast, the target protein potential of miR-4257 has not been previously reported. By searching the database, the ST13 tumor suppressor gene was identified as a candidate target 
gene of miR-4257. The expression of this gene is reported to be downregulated in colorectal cancer tissue suggesting that it is a candidate tumor suppressor gene (35). In the near future, we aim to clarify the functional activity of miR-4257 through this target gene.

In conclusion, the present study demonstrates that plasma exosomal miR-21 and miR-4257 show potential as biomarkers of recurrence in NSCLC patients. These biomarkers may be therapeutic targets in NSCLC.

\section{Acknowledgements}

The authors would like to thank Miss Junko Tamura (Department of Surgery, Teikyo University School of Medicine) for providing excellent technical assistance, and all members of the respiratory surgery group of Teikyo University School of Medicine.

\section{References}

1. Wakao F, Nishimoto H, Katanoda K, et al: Cancer Statistics in Japan 2015. Editorial Board of the Cancer Statistics in Japan (ed.). Foundation for Promotion of Cancer Research c/o National Cancer Center, Tokyo pp1-129, 2016.

2. Maeda T, Ueda H, Tabata M, Kiura K, Shibayama T, Gemba K, Takigawa N, Hiraki A, Katayama H and Harada M: Prognostic factors in advanced non-small cell lung cancer: Elevated serum levels of neuron specific enolase indicate poor prognosis. Jpn J Cin Oncol 30: 534-541, 2000.

3. Del Vescovo V, Grasso M, Barbareschi M and Denti MA: MicroRNAs as lung cancer biomarkers. World J Clin Oncol 5: 604-620, 2014

4. Wang QZ, Xu W, Habib N and Xu R: Potencial uses of microRNA in lung cancer diagnosis, prognosis, and theray. Curr Cnacer Drug Targets 9: 572-594, 2009.

5. Deng D, Liu Z and Du Y: Epigenetic alterations as cancer diagnostic, prognostic, and predictive biomatkers. Adv Genet 71 : 125-176, 2010.

6. Grady WM and Tewai M: The next thing in prognostic molecular markers: MicroRNA signatures of cancer. Gut 59: 706-708, 2010.

7. Chen X, Ba Y, Ma I, Cai X, Yin Y, Wang K, Guo J, Zhang Y, Chen J, Guo X, et al: Characteruzation of microRNAs in serum: A novel class of biomarkers for diagnosis of cancer and other diseases. Cell Res 18: 997-1006, 2008.

8. Heegaard NH, Schetter AJ, Welsh JA, Yoneda M, Bowman ED and Harris CC: Circulating micro-RNA expression profiles in early stage nonsmall cell lung cancer. Int J Cancer 130: $1378-1386,2012$

9. Ulivi P and Zoli W: miRNAs as non-invasive biomarkers for lung cancer diagnosis. Molecules 19: 8220-8237, 2014.

10. Powrózek T, Krawczyk P, Kowalski DM, Winiarczyk K, Olszyna-Serementa M and Milanowski J: Plasma circulating microRNA-944 and microRNA-3662 as potential histologic type-specific early lung cancer biomarkers. Transl Res 166: 315-323, 2015.

11. Zheng D, Haddadin S, Wang Y, Gu LQ, Perry MC, Freter CE and Wang MX: Plasma microRNAs as novel biomarker for early detection of lung cancer. Int J Clin Exp Pathol 4: 575-586, 2011.

12. Kowal J, Tkach M and Théry C: Biogenesis and secretion of exosome. Curr Opin Cell Biol 29: 116-125, 2014.

13. Ge Q, Zhou Y, Lu J, Bai Y, Xie X and Lu Z: miRNA in plasma exosome is stable under different storage conditions. Molecules 19: 1567-1575, 2014.

14. Palma J, Yaddannapudi SC, Pigati L, Havens MA, Jeong S, Weiner GA, Weimer KM, Stern B, Hastings ML and Duelli DM: MicroRNAs are exported from malignant cells in customized particles. Nucleic Acids Res 40: 9125-9138, 2012.

15. Simons M and Raposo G: Exosomes-vesicular carriers for intercelluar communication. Curr Opin Cell Biol 21: 575-581, 2009.
16. Peinado H, Alečković M, Lavotshkin S, Matei I, Costa-Silva B, Moreno-Bueno G, Hergueta-Redondo M, Williams C, García-Santos G, Ghajar C, et al: Melanoma exosomes educate bone marrow progenitor cells toward a pro-metastatic phenotype through MET. Nat Med 18: 883-891, 2012.

17. Valadi H, Ekström K, Bossios A, Sjöstrand M, Lee JJ and Lötvall JO: Exosome-mediated transfer of mRNAs and microRNAs is a novel mechanisms of genetic exchange between cells. Nat cell Bio 9: 654-659, 2007.

18. Hannafon BN and Ding WQ: Intercellular communication by exosome-derived microRNAs in cancer. Int J Mol Sci 14: 14240-14269, 2013.

19. Tanaka Y, Kamohara H, Kinoshita K, Kurashige J, Ishimoto T, Iwatsuki M, Watanabe M and Baba H: Clinical impact of serum exosomal microRNA-21 as a clinical biomarker in human esophageal squamous cell carcnoma. Cancer 119: 1159-1167, 2013.

20. Ogata-Kawata H, Izumiya M, Kurioka D, Honma Y, Yamada Y, Furuta K, Gunji T, Ohta H, Okamoto H and Sonoda H: Circulatung exosomal microRNAs as biomarkers of colon cancer. PLoS One 9: e92921, 2014.

21. Sugimachi K, Matsumura T, Hirata H, Uchi R, Ueda M, Ueo H, Shinden Y, Iguchi T, Eguchi H, Shirabe K, et al: Idenification of a bona fide microRNA biomarker in serum exosomes that predicts hepatocellular carcinoma recurrence after liver transplantation. Br J Cancer 112: 532-538, 2015.

22. Travis WD, Brambilla E, Buke AP, Marx A and Nicholson AG: WHO classification of tumors of the lung, pleura, thymus and heart. Bosman FT, Jaffe ES, Lakhani SR, Ohgaki H (eds): International Agency for Research on Cancer, France, pp1-pp412, 2015.

23. General rule for clinical and pathological record of lung cancer. The Japan Lung Cancer Society (ed.). Kenehara Co. Ltd, Tokyo, pp1-pp213, 2014.

24. Guidelines 2014 for treatment of lung cancer by EBM. Japan Lung Cancer Society (ed.) Kenehara Co. Ltd, Tokyo, pp1-pp201, 2014.

25. Livak KJ and Schmittgen TD: Analysis of relative gene expression data using real-time quantitative PCR and the 2(-Delta Delta C(T)) method. Methods 25: 402-408, 2001.

26. Zhang H, Li P, Ju H, Pesta M, Kulda V, Jin W, Cai M, Liu C, Wu H, $\mathrm{Xu}$ J, et al: Diagnostic and prognostic value of microRNA-21 in colorectal cancer: An original study and individual participant data meta-analysis. Cancer Epidemiol Biomarkers Prev 23: 2783-2792, 2014.

27. Wang ZX, Bian HB, Wang JR, Cheng ZX, Wang KM and De W: Prognostic significance of serum miRNA-21 expression in human non-small cell lung cancer. J Surg Oncol 104: 847-851, 2011.

28. Sanfiorenzo C, Iiie MI, Belaid A, Barlési F, Mouroux J, Marquette CH, Brest P and Hofman P: Two panels of plasma microRNAs as non-invasive biomarkers for prediction of recurrence in resectable NSCLC. PLoS One 8: e54596, 2013.

29. Gao F, Chang J, Wang H and Ahang G: Potential diagnostic value of miR-155 in serum from lung adenocarcinoma patients. Oncol Rep 31: 351-357, 2014.

30. Liu XG, Zhu WY, Huang YY, Ma LN, Zhou SQ, Wang YK, Zeng F, Zhou JH and Zhang YK: High expression of serum miR-21 and tumor miR-200c associated with poor prognosis in patients with lung cancer. Med Oncol 29: 618-626, 2012.

31. Esquela-Kerscher A and Slack FJ: Oncomirs-microRNAs with a role in cancer. Nat Rev Cancer 6: 259-269, 2006.

32. Meng F, Henson R, Wehbe-Janek H, Ghoshal K, Jacob ST and Patel T: MicroRNA-21 regulates expression of the PTEN tumor suppressor gene in human hepatocellular cancer. Gastroenterology 133: 647-658, 2007.

33. Motoyama K, Onoue H, Mimori K, Tanaka F, Kojima K, Uetake H, Sugihara K and Mori M: Clinicopathological and prognostic significance of pded4 and microRNA-21 in human gastric cancer. Int J Oncol 36: 1089-1095, 2010.

34. Horiuchi A, Iinuma H, Akahane T, Shimada R and Watanabe T: Prognostic significance of PDCD4 expression and association with microRNA-21 in each Dukes' stage of colorectal cancer patients. Oncol Rep 27: 1384-1392, 2012.

35. Bai R, Shi Z, Zhang JW, Li D, Zhu YL and Zheng S: ST13, a proliferation regulator, inhibits growth and migration of colorectal cancer cell lines. J Zhejiang Univ Sci B 13: 884-893, 2012. 